\title{
Diagnosis of interstitial cystitis
}

\author{
Lesley K. Carr, MD, FRCSC;* Jacques Corcos, MD; ; J. Curtis Nickel, MD, FRCSC; \\ Joel Teichman, MD, FRCSC ${ }^{\mathcal{S}}$
}

Can Urol Assoc J 2009;3(1):81-6

\section{Methodology}

The following guidelines were based on consensus conference proceedings (Table 1). For each section, a MEDLINE search of the English language literature was undertaken and scrutinized.

\section{Terminology}

There is current controversy over the optimal name for the condition classically known as interstitial cystitis (IC). The term IC reflects the clinical interpretation of the symptom of pain centred in the region of the bladder and implies local inflammation, which is often not demonstrable. In 2002, the International Continence Society defined painful bladder syndrome (PBS) as the complaint of suprapubic pain related to bladder filling, accompanied by other symptoms such as increased daytime and nighttime frequency, in the absence of proven urinary infection or other obvious pathology. 'Subsequent to this definition, some have used the term IC to reflect patients who meet the classic National Institutes of Health (NIH) criteria ${ }^{2}$ and PBS to reflect the group that may have identical symptoms but have not undergone formal hydrodistension or do not meet all of the NIH criteria. It is recognized that the $\mathrm{NIH}$ criteria were designed to delineate a homogeneous population for research trials and are overly restrictive for use in routine clinical practice. ${ }^{3}$ More recently, because of similarities with IC/PBS and other chronic pain syndromes, there has been a move to change terminology to reflect widely encompassing pain taxonomies. This is reflected in the term bladder pain syndrome. Thus at the moment, all 3 existing English terminologies (IC, PBS or bladder pain syndrome) are used worldwide. The corresponding French terminology is Cystite Interstitielle, Cystalgie a Urine Claire or Cystalgie Abacterienne. Hopefully, the next decade will see widespread adoption of a single name and definition.

\section{History, frequency volume chart and physical examination}

A thorough general medical history is of paramount importance to identify typical diagnostic

Table 1. Guidelines for evaluation of interstitial cystitis painful bladder syndrome summation

\begin{tabular}{llcc}
\hline Guideline & \multicolumn{1}{c}{ Evaluation } & $\begin{array}{c}\text { Level of } \\
\text { evidence }\end{array}$ & $\begin{array}{c}\text { Grade of } \\
\text { recommendation }\end{array}$ \\
\hline Recommended & & 4 & C \\
& History & \\
& $\begin{array}{l}\text { Physical examination } \\
\text { (abdomen, back, } \\
\text { pelvic, rectal, } \\
\text { musculoskeletal, } \\
\text { focused-neuro) }\end{array}$ & \\
& Frequency volume \\
& chart \\
& Symptom score (PUF, \\
& ICSI/ICPI, UW-IC) \\
& Urine (urinalysis, \\
& culture, cytology)
\end{tabular}

Optional $2-3$

C

(recommended under certain

circumstances)

Cystoscopy
Cystoscopy with
hydrodistension
(general or intravesical
anesthetic)
Potassium
sensitivity test
Alkalinized lidocaine
challenge

Not recommended (unless specific unusual concerns)

Bladder biopsy Urodynamics

$I C P I=$ Interstitial Cystitis Problem Index; ICSI = Interstitial Cystitis Symptom Index: PUF = Pain Urgency and Frequency; UW-IC = Wisconsin Interstitial Cystitis. 
symptoms of IC/PBS and other potential mimicking causative conditions. Storage and voiding symptoms along with pain characteristics should be elicited. The location of pain, relation to bladder filling/emptying or other triggers such as diet, intercourse or menses; duration and a description of the type of pain can all be useful. In early or milder IC/PBS, patients may not describe frank pain, but rather just pressure or an uncomfortable sensation of having to urinate. Despite the absence of urinary tract infection (UTI) being a prerequisite at the time of diagnosis, up to $50 \%$ of patients will have a previous history of UTI. A past history of pelvic surgery or radiation, medications that can cause cystitis, autoimmune diseases, other gynecological or bowel symptoms can be important.

A frequency volume chart is advocated to differentiate polyuria from the classic small voided volumes expected with IC/PBS. This also helps to determine the severity of the storage symptoms and can be used for positive reinforcement related to behavioural and pharmacological intervention.

Although a complete physical examination should be performed, particular focus is directed to looking for bladder distension, hernias, signs of nerve entrapment and trigger points abdominally. A musculoskeletal and focused neurological examination may also be contributory. A digital rectal examination (DRE) in men and pelvic examination in women is essential. For the DRE, prostate characteristics should be elicited along with discrete point tenderness of the prostate and pelvic floor muscles. Prostatic massage could be considered if pain appears to be more related to the prostate. The female pelvic examination should screen for vulvodynia, vaginitis, atrophic changes, prolapse, cervical pathology, adnexal masses or tenderness, pelvic floor muscle tenderness, weakness and spasm, and point tenderness over the urethra or bladder.

Abdominal or pelvic ultrasonography, or other imaging modality may be useful when alternative clinical conditions are questioned but are expected to be normal if IC/PBS is the only diagnosis.

\section{Laboratory tests}

A urine dipstick represents the minimum required laboratory test. Glucose, leukocytes, hematuria, nitrites and osmolality may be simply screened for. If signs of UTI are identified, a culture and sensitivity is required and possibly testing for acidfast bacilli if sterile pyuria persists. A cytology is indicated if microscopic hematuria is identified or if there are other risk factors for urothelial carcinoma present. Hematuria has been reported in up to $41 \%$ of patients with IC/PBS (only $2 / 60$ were gross hematuria) and none were associated with a life-threatening urological condition. ${ }^{4}$

\section{Symptom scores (recommended)}

Symptom scores for IC/PBS could have theoretical utility in diagnosis, grading severity of disease or tracking response to therapeutic intervention. Four self-administered symptom scores for IC/PBS have been suggested and assessed to variable extents: the Interstitial Cystitis Symptom Index (ICSI), ${ }^{5}$ the Interstitial Cystitis Problem Index $(\mathrm{ICPI}),{ }^{5}$ the Wisconsin Interstitial Cystitis Scale (UW-IC Scale $)^{6}$ and the Pain, Urgency and Frequency (PUF) score. ${ }^{7}$

The first symptom score, the UW-IC Scale, contained 7 questions directly related to bladder symptoms in the previous 24 hours and a control of 18 non-bladder related items referred to as reference items. ${ }^{6}$ The original article showed content and construct validity and reliability, but was based on only 8 IC patients and 9 controls. ${ }^{6}$ Further studies have re-evaluated the instrument in larger series of IC patients. ${ }^{8-10}$ Goin and colleagues ${ }^{8}$ used the UW-IC Scale in 30 women with IC enrolled in a phase 2 trial of intravesical BCG. They found that the psychometric properties were similar to standards of other instruments used in clinical research, but also suggested that the pelvic item from the reference items should be included with the IC items and that a possible ceiling effect for pain may lower the sensitivity of the UW-IC Scale for detecting differences in intervention outcomes. ${ }^{8}$ The UW-IC Scale was also shown to correlate well with the ICSI and ICPI in 107 patients with IC. ${ }^{9}$ In addition, it has demonstrated responsiveness to change over time in patients with IC and was recommended as a secondary end point in clinical trials. ${ }^{10}$

The ICSI and ICPI (also known as the O'Leary-Sant Interstitial Cystitis Symptom and Problem Index) were created after validation in a group of 45 IC patients and 67 gynecological 
controls. ${ }^{5}$ The process began with a 23 -item index, and analysis suggested a 4-item symptom and problem index focusing on urgency, frequency, nocturia and pain over the past month, met standards for variability, test retest reliability, internal consistency and construct validity. ${ }^{5}$ Lubeck and colleagues ${ }^{11}$ assessed the utility of the ICSI in 376 patients with IC enrolled in a trial of varying doses of pentosan polysulfate sodium. They also found the ICSI to be a valid, reliable and responsive measure of change in IC symptoms. ${ }^{11}$ As with the UW-IC Scale, Propert and coauthors ${ }^{10}$ demonstrated that the ICSI and ICPI were both responsive to change in patients with IC. An interesting finding by Diggs and colleagues ${ }^{12}$ was that the ICSI underestimated the degree of urgency in IC patients compared with questioning following the International Continence Society definition.

The final symptom score for IC is the PUF score. ${ }^{7}$ This instrument does not appear to have been subject to as extensive a validation process as the other 3 instruments and has additional items related to pelvic pain and pain related to sexual intercourse. Three hundred and thirtyfour patients from urology or gynecology clinics underwent a potassium sensitivity test (PST) and PUF score. Seventy-six percent of patients with PUF scores of 15-19 had positive PSTs.? The PUF score was found to be accurate with the PST as the reference standard and reproducibility was found to be accurate in a subset of 32 women. ${ }^{7}$ Kushner and Moldwin ${ }^{13}$ examined the ability of the PUF score and the ICSI/ICPI to distinguish IC from other urinary tract pathologies in a group of 220 patients at a urology clinic. All 3 scales did distinguish IC, and the PUF score $(\geq 13)$ did so more efficiently. However, none of the tools had sufficient specificity to serve as a sole diagnostic indicator. ${ }^{13}$

Based on current literature, we recommend the use of the ICSI, ICPI, UW-IC Scale, or PUF Score to assist with diagnosis and follow response to therapeutic intervention in patients with IC.

\section{Potassium sensitivity test (optional)}

A potassium chloride bladder permeability test has been suggested to assist with the diagnosis of IC and, in particular, to identify a subset that may have "dysfunctional epithelium" (deficient glycosaminoglycan [GAG] layer ${ }^{14}$ ). The technique, comparing subjective pain or urgency responses to intravesically instilled $0.4 \mathrm{M}$ potassium chloride and water, was described by Parsons and colleagues ${ }^{14}$ but has been modified by other authors, including variants such as the comparative cystometrogram test. ${ }^{15} \mathrm{~A}$ positive potassium sensitivity test normalizes with successful epithelial directed therapy. ${ }^{16}$ In theory, it would be an advantage to guiding management if a positive potassium sensitivity test correlated well with a favourable response to agents that attempt to replenish the GAG layer, as has been suggested in 2 small series. ${ }^{17,18}$

The sensitivity and specificity of this test $(69.5 \%$ and $50 \%$ ) were found by Chambers and colleagues ${ }^{19}$ to be poor, adding no additional utility over history and cystoscopy. However, their modification of the test used a different, nonvalidated scale and assessed only pain and not urgency responses when compared with the original description of the test. Others have found that the potassium sensitivity test did not correlate with either cystoscopic findings or bladder capacity on urodynamics. ${ }^{20}$ However, these conclusions more underscore the challenges faced in diagnosis of IC because none of our other available tests including cystoscopy, biopsy or urodynamics correlate well with symptoms or represent ideal benchmarks for comparison.

At this point, the use of the potassium sensitivity test has not been widely validated and the ability of this test to predict the outcome with GAG replenishing therapies has only been suggested by 2 small series. For these reasons, the potassium sensitivity test is not recommended as a standard evaluation for IC, but can be considered an optional adjunctive evaluation. For patients in whom the diagnosis is early or confusing, the provocative potassium sensitivity test may provide supporting evidence for diagnosis.

\section{Cystoscopy local anesthetic (optional)}

Cystoscopy performed alone without hydrodistension is expected to be normal (except for discomfort and reduced "functional" bladder capacity) in most patients with IC/PBS. Hunner ulcers or patches are found in about $6 \%-8 \%{ }^{21}$ and are associated with more severe symptoms, and reduced urodynamic and anesthetic capacity. ${ }^{22,23}$ 
The classic findings of terminal hematuria and glomerulations are reliably identified only after a formal hydrodistension under anesthetic. As such, the purpose of cystoscopy alone should only be viewed as a tool to rule out bladder cancer/ carcinoma in situ, to identify Hunner ulcers that reflect severe disease (information that may impact treatment decisions), to determine effect on pelvic pain during bladder filling and emptying, to objectively evaluate "functional" bladder capacity, to facilitate appropriate pelvic examination and to reassure the patient. The incidence of bladder cancer presenting with symptoms compatible with IC/PBS is rare. Tissot and colleagues ${ }^{24}$ found $1 \%$ of 600 patients referred with a diagnosis of IC/PBS had bladder cancer. These patients were likely a highly selected cohort perhaps representing a worst case scenario. Most (5/6) with cancer were older than 60 and all except 2 had microscopic hematuria or positive cytology.

Cystoscopy is not mandatory in a young woman with symptoms of IC/PBS and no risk factors for bladder cancer or other pelvic conditions. This may enable nonurologist physicians to initiate treatment earlier in the stage of disease when it is possibly more effective. ${ }^{25}$ It is reasonable to employ cystoscopy to assist in making a diagnosis before initiating therapy, especially if there is any indication on history, physical examination, urinalysis or cytology that other disease needs to be ruled out (usual case).

\section{Hydrodistension (optional)}

Hydrodistension under general anesthetic allows for stratification of patients into those with more "classic" disease associated with ulcers and glomerulations from those with with no obvious mucosal abnormalies. ${ }^{2}$ The technique of diagnostic hydrodistension generally involves gravity filling of the bladder at $70-100 \mathrm{~cm} \mathrm{H}_{2} \mathrm{O}$ for a minimum of 2 minutes performed under general or regional anesthetic. Maximum anesthetic capacity is determined whereby the inflow backs up in the drip chamber or leakage occurs per urethra despite compression against the cystoscope. Although severely reduced anesthetic bladder capacities $(<400 \mathrm{~mL})$ do correlate with pain, ${ }^{23}$ more than $50 \%$ of patients with IC/PBS show capacities more than $800 \mathrm{~mL} .^{23}$ The presence of terminal hematuria on draining the infusion fluid and the appearance of petechial submucosal hemorrhages (glomerulations) has been suggested to be characteristic of IC and one of the prerequisite findings in the National Institute of Diabetes and Digestive and Kidney Diseases (NIDDK) criteria. ${ }^{2}$ Glomerulation severity has also been graded. ${ }^{2}$ A possible relationship between glomerulations and angiogenic growth factors has been found suggesting that these growth factors may have an important role in the pathogenesis of IC/PBS. ${ }^{26}$

Despite the initial adoption of the hydrodistension findings of glomerulations as a criteria for the diagnosis of IC by the NIH, about $8 \%$ with a diagnosis of IC/PBS do not show glomerulations. ${ }^{22,27}$ The severity of glomerulations was found to correlate poorly with symptoms and with histological evidence of inflammation. ${ }^{28}$ In contrast, Lamale and colleagues ${ }^{29}$ found strong correlation with pain and hydrodistension findings. Their series was small (12) including perhaps more severe patients as evidenced by a mean anesthetic bladder capacity of $604 \mathrm{~mL}$, but represented an untreated cohort where they postulated there were no confounders of treatment allowing a true correlation to be identified. In another series of 84 patients, cystoscopy with hydrodistension provided little useful information above and beyond the history and physical examination findings. ${ }^{27}$ Additionally, the specificity of glomerulations was brought into question when Waxman and colleagues ${ }^{30}$ found characteristic glomerulations in $45 \%$ of 20 normal women who consented to undergo hydrodistension at the time of tubal ligation.

Although relief of symptoms does occur in some patients and is a helpful clinical sign, the symptom relief is typically short lived ${ }^{27,31}$ and thus as a repetitive treatment modality, hydrodistension is probably not worth the potential morbidity and cost associated with repeated general anesthetics. Employing an alkalinized lidocaine solution, ${ }^{32}$ a modified hydrodistension can be performed under local anesthetic. Such an approach is inexpensive, safe and may give similar information regarding mucosal integrity and clinical response as that obtained from the more formal hydrodistension performed under general anesthetic.

Cystoscopy under general or regional anesthetic with hydrodistension is considered optional for routine evaluation of patients being diagnosed with IC/PBS. 


\section{Bladder biopsy (not recommended)}

There are no specific features found on bladder biopsy to confirm a diagnosis of IC/PBS. Findings related to chronic inflammation are not specific, overlapping with other etiologies, and they correlate poorly to cystoscopic findings observed during hydrodistension. ${ }^{28}$ Between $30 \%{ }^{28}$ and $43 \%{ }^{33}$ of patients with a clinical diagnosis of IC/PBS may have normal histology. Thus the use of biopsy to confirm or refute diagnosis in IC/PBS has not been validated.

However, correlations have been found with specific types of pathological findings and symptoms. For example, mucosal denudation (i.e., Hunner ulcer) and submucosal hemorrhage were highly associated with pain, and mast cell count on tryptase stain, complete loss of urothelium (i.e., Hunner ulcer), granulation tissue in the lamina propria, and vascular density were associated with nocturia in a multivariate analysis of patients from the IC database. ${ }^{34}$ The importance of mast cells has been controversial. Dundore and colleagues ${ }^{35}$ found no significant difference in mast cell counts in the lamina propria or detrusor on Giemsa stained sections between IC/PBS patients compared with controls. Because an association between specific pathological features and symptoms may exist, it is reasonable to include a bladder biopsy and pathological classification in future research studies of natural history or therapeutic intervention.

When a biopsy is indicated for research or to rule out carcinoma in situ if suspected by a focal lesion or abnormal cytology, this should be performed from the most abnormal appearing area ${ }^{36}$ and should follow hydrodistension to avoid increased risk of bladder perforation.

Routine bladder biopsies are not recommended for the diagnosis of IC/PBS but may be considered in research trials or to rule out other specific diagnosis such as carcinoma in situ when clinically indicated.

\section{Urodynamics (not recommended)}

Filling cystometry (CMG) has been advocated by some in the diagnosis of IC/PBS., ${ }^{2,37}$ Certainly there is overlap between the conditions of overactive bladder (OAB) dry and symptoms of IC/PBS and the finding of detrusor overactivity (DO) on filling
CMG may lead the clinician to initiate therapy with anticholinergic agents. However, urodynamics (UDS) may miss DO in up to $50 \%$ of patients compared with ambulatory UDS $^{38}$ and as a result most clinicians are accustomed to using anticholinergic agents for symptoms of OAB without first performing UDS.

According to the NIDDK criteria, the finding of a capacity greater than $350 \mathrm{~mL}$, first sensation of having to void greater than $150 \mathrm{~mL}$, or the presence of DO are exclusionary for a diagnosis of classic IC. ${ }^{2}$ However, it is recognized that about $15 \%$ of patients diagnosed with IC/PBS will demonstrate $\mathrm{DO}^{39}$ and thus the coexistence of urge incontinence or DO should not preclude a diagnosis of IC/PBS. Other findings on UDS from the IC Database Study were a reduced first sensation to void (mean 81, standard deviation [SD] 64, mL) and maximum sensory capacity (mean 198, SD $107 \mathrm{~mL}$ ). ${ }^{23}$ Although these UDS parameters do correlate well with frequency, nocturia and urgency, ${ }^{39}$ they have not been well correlated with global pain, ${ }^{39}$ cystoscopic findings at hydrodistension, other than the presence of a Hunner ulcer, or results of therapeutic intervention. ${ }^{5}$ Bladder capacity may be assessed less invasively and more cost-effectively by means of a frequency volume chart with self-measurement of voided volumes and this has been shown to correlate with maximum cystometrogam capacity and first sensation of having to void in patients with IC/PBS. ${ }^{39}$ If a cystoscopy under local anesthetic is planned, a functional bladder capacity and its relation to the patient's pain can be assessed with the patient awake.

The use of a comparative cystometrogram test (CCT) employing normal saline versus $0.3 \mathrm{M}$ potassium chloride has been suggested as a diagnostic tool for IC/PBS. ${ }^{15}$ However, Phillip and colleagues ${ }^{40}$ found a more pronounced reduction in maximum CMG capacity with potassium chloride in the detrusor overactivity group compared with the IC group, suggesting a poor positive predictive value for the CCT in distinguishing IC from DO.

Pressure flow studies with or without electromyography may be useful in some situations where there are coexistent voiding symptoms with suspicion of bladder outlet obstruction or voiding dysfunction due to high tone pelvic floor dysfunction.

Urodynamics studies are not recommended 
Carr et al.

in the standard diagnostic evaluation of a patient suspected of having IC/PBS.

Consensus panel: *Chair; Assistant Professor, Department of Surgery, University of Toronto, Sunnybrook Health Sciences Centre, Toronto, Ont; † Member; Professor of Urology, McGill University, Jewish General Hospital, Montréal, Que.; † Member; Professor of Urology, Queen's University, Toronto, Ont.; §Member; Associate Professor, University of British Columbia, Vancouver, BC

This article has been peer reviewed.

Competing interests: Dr. Carr is on the Canadian advisory board for JanssenOrtho, Pfizer, Astellas, Allergan and Gynecare. She was a lecturer for CE events with financial support from Janssen-Ortho, Pfizer, Allergan and Triton. She received support for a surgical preceptorship from Allergan, Gynecare and Mentor/Coloplast. She received support for research from Stellar Labs, the National Institute of Health and Cook Myosite.

\section{References}

1. Abrams P, Cardozo L, Fall M, et al. The standardization of terminology of lower urinary tract function: report from the Standardisation Sub-committee of the International Continence Society. Urology 2003;61:37-49.

2. Gillenwater JY, Wein AJ. Summary of the NIDDK workshop on interstitial cystitis, NIH, Bethesda, MD, August 28-29, 1997. J Urol 1988; 140:203-6.

3. Hanno PM, Landis JR, Matthews-Cook Y, et al. The diagnosis of interstitial cystitis revisited: lessons learned from the national instititutes of health interstitial cystitis database study. J Urol 1999;161:553-7.

4. Gomes CM, Sanchez-Ortiz RF, Harris C, et al. Significance of hematuria in patients with interstitial cystitis: review of radiographic and endoscopic findings. Urology 2001;57:262-5.

5. O'Leary MP, Sant Gr, Fowler FJ, et al. The interstitial cystitis symptom index and problem index. Urology 1997;49(5A Suppl):58-63.

6. Keller ML, McCarthy DO, Neider RS. Measurement of symptoms of interstitial cystitis. A Pilot Study. Urol Clin North Am 1994;21:67-71.

7. Parsons CL, Dell J, Stanford EJ, et al. Increased prevalence of interstitial cystitis: previously unrecognized urologic and gynecologic cases identified using a new symptom questionnaire and intravesical potassium sensitivity. Urology 2002;60:573-8.

8. Goin JE, Olaleye D, Peters KM, et al. Psychometric analysisi of the University of Wisconsin interstitial cystitis scale: implications for use in randomized clinical trials. J Urol 1998; 159:1085-90

9. Sirinian E, Azevedo K, Payne CK. Correlation between 2 interstitial cystitis symptom instruments. J Urol 2005;173:835-40.

10. Propert KJ, Mayer RD, Wang Y, et al. Responsiveness of symptom scales for interstitial cystitis. Urology 2006;67:55-9.

11. Lubeck DP, Whitmore K, Sant GR, et al. Psychometric validation of the O'Leary-Sant interstitial cystitis symptom index in a clinical trial of pentosan polysulfate socium. Urology 2001;57:62-6.

12. Diggs C, Meyer WA, Langenberg P, et al. Assessing urgency in interstitial cystitis/painful bladder syndrome. Urology 2007;69:210-4.

13. Kushner L, Moldwin RM. Efficiency of questionnaires used to screen for interstitial cystitis. J Urol 2006; 176:587-92.

14. Parsons $\mathrm{CL}$, Greenberger $\mathrm{M}$, Gabal $\mathrm{L}$, et al. The role of urinary potassium in the pathogenesis and diagnosis of interstitial cystitis. J Urol 1998;159:1862-6.

15. Daha LK, Riedl CR, Hohlbrugger $G$, et al. Comparative assessment of maximal bladder capacity, $0.9 \% \mathrm{NaCl}$ versus $0.2 \mathrm{M} \mathrm{KCl}$, for the diagnosis of interstitial cystitis: a prospective controlled study. J Urol 2003; 170:807-9.

16. Parsons CL, Forrest J, Nickel JC, et al. Effect of pentosan polysulfate therapy on intravesical potassium sensitivity. Urology 2002;59:329-33.
17. Gupta SK, Pidcock L, Parr NJ. The potassium sensitivity test: a predictor of treatment response in interstitital cystitis. BJU Int 2005;96:1063-6.

18. Teichman JMH, Nielsen-Omeis BJ. Potassium leak test predicts outcome in interstitial cysititis. J Urol 1999;161:1791-6.

19. Chambers GK, Fenster HN, Cripps $S$, et al. An assessment of the use of intravesical potassium in the diagnosis of intersititial cystitis. J Urol 1999;162:699-701.

20. Gregoire $M$, Liandier $F$, Naud A, et al. Does the potassium stimulation test predict cystometric cystoscopic outcome in intersititital cystitis? J Urol 2002;168:556-7.

21. Parsons CL, Walker CJ. Cystoscopic changes in interstitial cystitis. Urology 1996;48: 289-90.

22. Messing E, Pauk D, Schaeffer A, et al. Associations among cystoscopic findings and symptoms and physical examination findings in women enrolled in the interstitial cystitis data base (ICDB) study. Urology 1997;49:81-5.

23. Nigro DA, Wein AJ, Foy M, et al. Associations among cystoscopic and urodynamic findings for women enrolled in the interstitial cystitis data base (ICDB) study. Urology 1997:49:86-92.

24. Tissot WD, Kiokno AC, Peters KM. A referral center's experience with transitional cell carcinoma misdiagnosed as interstitial cystitis. J Urol 2004;172:478-80.

25. Forrest JB, Sebastyanski $P, O^{\prime}$ Brien-Westbrook $M$. Observations on the clinical factors affecting the treatment outcomes of interstitial cystitis. Poster presented at: International Pelvic Pain Society (IPPS) Annual Meeting; August 5-7, 2004; Chicago, III.

26. Tamaki $M$, Saito R, Ogawa 0 , et al. Possible mechanisms inducing glomerulations in interstitial cystitis: relationship between endoscopic findings and expression of angiogenic growth factors. J Urol 2004;172:945-8.

27. Ottem DP, Teichman JMH. What is the value of cystoscopy with hydrodistension for interstitial cystitis? Urology 2005;66:494-9.

28. Denson MA, Griebling TL, Cohen MB, et al. Comparison of cystoscopic and histological findings in patients with suspected interstitial cystitis. J Urol 2000;164:1908-11.

29. Lamale LM, Lutgendorf SK, Hoffman AN, et al. Symptoms and cystoscopic findings in patients with untreated interstitial cystitis. Urology 2006;67:242-5.

30. Waxman JA, Sulak PJ, Kuehl TJ. Cystoscopic findings consistent with interstitial cystitis in normal women undergoing tubal ligation. J Urol 1998;160:1663-7.

31. Cole EE, Scarpero HM, Dmochowski RR. Are patient symptoms predictive of the diagnostic and/or therapeutic value of hydrodistension? Neurourol Urodyn 2005;24:638-42.

32. Henry R, Patterson L, Avery N, et al. Absorption of alkalinized intravesical lidocaine in normal and inflamed bladdrs: A simple method to improve bladder anesthesia. J Urol 2001;165:1900-3.

33. Matilla J. Vascular immunopathology in interstitial cystitis. Clin Immunol Immunopathol 1992;23:648-55.

34. Tomaszewski JE, Landis JR, Russack V, et al. Biopsy features are associated withprimary symptoms in interstitial cystitis: results from the interstitial cystitis database study. Urology 2001;57:67-81.

35. Dundore PA, Schwartz AM, Semerijan H. Mast cell counts are not useful in the diagnosis of nonulcerative interstitial cysititis. J Urol 1996;155:885-7.

36. Rosamilia A, Igawa Y, Higashi S. Pathology of interstitial cystitis. Int J Urol 2003;10:11-5.

37. Pontari MA, Hanno PM, Wein AJ. Logical and systematic approach to the evaluation and management of patients suspected of having interstitial cystitis. Urology 1997:49:114-20.

38. Radley SC, Rosario DJ, Chapple CR, et al. Conventional and ambulatory urodynamic findings in women with symptoms suggestive of bladder overactivity. J Urol 2001;166:2253-8.

39. Kirkemo A, Peabody M, Diokno AC, et al. Associations among urodynamic findings and symptoms in women enrolled in the Interstitial Cystitis Dta Base (ICDB) study. Urology 1997; 49:76-80.

40. Phillip J, Willmott S, Irwin P. Interstitial cystitis versus detrusor overactivitiy: a comparative randomized, controlled study of cystometry using saline and $0.3 \mathrm{M}$ potassium chloride. J Urol 2006; 175:566-70.

Correspondence: Dr. Lesley Carr, Sunnybrook Health Science Centre, University of Toronto, 2075 Bayview Ave., Toronto ON M4N 3M5; lesley.car@@sunnybrook.co 